\title{
Criminologie
}

\section{Éditorial : criminalités économiques}

\section{Jean-Luc Bacher}

Volume 30, numéro 1, printemps 1997

Criminalités économiques

URI : https://id.erudit.org/iderudit/017394ar

DOI : https://doi.org/10.7202/017394ar

Aller au sommaire du numéro

Éditeur(s)

Les Presses de l'Université de Montréal

ISSN

0316-0041 (imprimé)

1492-1367 (numérique)

Découvrir la revue

Citer ce document

Bacher, J.-L. (1997). Éditorial : criminalités économiques. Criminologie, 30(1),

3-8. https://doi.org/10.7202/017394ar d'utilisation que vous pouvez consulter en ligne.

https://apropos.erudit.org/fr/usagers/politique-dutilisation/ 
Criminalités économiques. Voilà un thème qui ne manquera pas de rappeler à certains de nos lecteurs la parution, il y a vingt ans cette année, d'un numéro de la revue qui s'intitulait alors Affaires et criminalité au Québec.

Criminalités économiques au pluriel, car les écrits rassemblés dans ce numéro rendent compte de formes de délinquance qui, si elles s'inscrivent toutes dans le cadre de relations ou activités économiques, sont assez différentes les unes des autres.

Cette nouvelle incursion dans le monde de la délinquance économique nous situe bien évidemment très loin de la criminalité sulfureuse et violente que les médias à sensations aiment à relater. Elle nous fait notamment pénétrer dans le monde des échanges commerciaux.

Si ce monde semble jouir aujourd'hui d'une certaine considération dans le public, il a autrefois été perçu comme peu recommandable et mal famé. Mercure n'était-il pas à la fois le dieu des voleurs et des marchands ? Ce père de la démocratie occidentale, qu'est Aristote voyait dans le commerce une activité que l'on ne pouvait exercer sans manquer aux exigences de la justice, une activité « qui n'a point de terme où puisse s'arrêter la cupidité' ». Pour le philosophe, le commerçant était à ce point indigne qu'il ne trouvait pas judicieux de lui reconnaître les droits et responsabilités politiques incombant, dans la cité, aux citoyens libres. Si les propos d'Aristote peuvent sembler quelque peu excessifs, ils nous incitent toujours à nous intéresser au monde des activités économiques afin de chercher à savoir si ce monde est plus criminogène que les autres et, dans l'affirmative, dans quelle mesure.

Parler de criminalité économique, c'est consentir à porter son attention sur d'autres délinquants que ceux qui suscitent dans le public toute la gamme des sentiments se situant entre la haine et la pitié. C'est aussi se pencher sur d'autres délinquants que ceux qui inspirent aux professionnels de l'intervention leurs désirs d'aider, d'éduquer, de socialiser, de réintégrer, de soigner, bref de traiter.

Dans le spectre des phénomènes criminels, la criminalité économique se situe à cette extrémité où la majeure partie des comportements délinquants sont, à l'instar d'innombrables comportements respectables, motivés par l'envie de s'enrichir, par l'aspiration à plus de bien-être, par le goût d'un

1. ARISTOTE, La Politique, Paris, Denoël/Gonthier, P.U.F.. 1980, p. 32. 
certain luxe, par le souci d'améliorer ses conditions matérielles ou par la quête de plus de sécurité.

Des auteurs de ces crimes, il est requis plus de qualités que de tares : il leur faut notamment de l'imagination, de l'ingéniosité, des connaissances techniques (informatiques, bancaires, commerciales, etc.), du savoir-faire, de l'expérience et pas mal d'intelligence pour réaliser leurs crimes.

Plutôt que d'attirer sur eux du mépris, de la pitié, de la haine ou de la condescendance, les délinquants économiques suscitent souvent dans leur entourage ou dans le public de la compréhension et de la complaisance. Certains crimes économiques valent même à leurs auteurs de recevoir des signes d'approbation et d'admiration. Quant aux traitements qu'il serait envisageable de proposer aux délinquants économiques, ils ne semblent pas répondre à un besoin impérieux. Loin s'en faut.

La criminalité économique demeure donc d'autant moins connue qu'elle est peu spectaculaire, peu menaçante, difficile à saisir et donc d'autant moins dérangeante. Cela dit, il est vrai que cette criminalité, qui a naguère fait les manchettes n'est plus aujourd'hui sous les feux de la rampe. Il peut donc sembler moins évident d'en parler maintenant qu'il y a vingt ans. À l'époque, la criminalité en col blanc se voyait vouer beaucoup d'attention de la part de l'ONU et du Conseil de l'Europe ${ }^{2}$. Elle faisait grandement parler d'elle tout aussi bien dans les milieux scientifiques que dans ceux de la politique et des médias d'information.

La criminalité économique, qu'elle soit ou non imputable aux milieux d'affaires, reste toutefois un phénomène aussi vaste que réel. Dans ses formes les plus bénignes, elle est le fait d'un nombre considérable d'individus et d'entreprises. Il suffit de songer à la fraude fiscale ou à la fraude à I'assurance. Dans ses formes les plus aiguës, elle porte parfois sur des sommes énormes.

Sous un angle rigoureusement juridique, la criminalité économique est un domaine en expansion, puisque de nouvelles infractions ont encore été introduites, ces dernières années, dans l'arsenal des interdits pénaux occidentaux. Que l'on songe notamment au délit d'initiés.

Bien qu'étant moins « au goût du jour » qu'il y a vingt ans, la criminalité économique continue de susciter un intérêt certain dans les milieux scientifiques et gouvernementaux. À vrai dire, il s'agit d'un intérêt plus sélectif

2. J. RICO, in Affaires et criminalité au Québec, Criminologie, Montréal, P.U.M., 1977 , p. 5. 
qu'autrefois, en ce sens que les décideurs et les chercheurs contemporains s'attaquent plutôt à des formes ou aspects particuliers de cette criminalité qu'au phénomène dans son ensemble. Pensons, par exemple, aux efforts scientifiques et politiques déployés ces dernières années sur les problèmes liés au blanchiment d'argent et à la corruption. C'est ainsi que le dernier congrès de la Société de défense sociale, en novembre 1996, fut consacré à la corruption.

La criminalité économique est un vaste phénomène polymorphe dont l'ampleur des effets est d'une portée sociale importante. En effet, outre qu'elle affecte dans leurs intérêts économiques un nombre considérable d'assurés, de contribuables, de consommateurs ou d'agents économiques de toutes sortes, elle est ultimement de nature à occasionner des dommages moraux ou immatériels quand, comme c'est notamment le cas de la fraude fiscale ou, dans certains pays, de la corruption, elle est réputée très répandue et peu réprimée. Ainsi elle érode les liens de confiance et de loyauté dont dépend directement la survivance des grands contrats juridiques et politiques sur lesquels est fondée l'organisation sociale.

La criminalité économique reste d'autant plus digne d'intérêt que de nombreuses questions relatives aux réactions à lui opposer restent âprement débattues. On peut citer par exemple la nature des peines devant être infligées aux entreprises. Cette criminalité requiert que soient élaborées des politiques criminelles originales, à la réalisation desquelles les secteurs privé et public sont condamnés à collaborer si l'on entend déployer contre elle des stratégies un tant soit peu cohérentes et fonctionnelles. Ici nous songeons prioritairement à la mise en œuvre de moyens de prévention et de détection du crime. Une telle collaboration semble d'ailleurs de plus en plus incontournable en cette époque où la demande en sécurités et protections de toutes sortes va en augmentant de façon importante.

Les contributions réunies sous le thème du présent numéro sont au nombre de quatre. Que l'on n'y voie pas l'incidence de consignes rédactionnelles nouvelles ni l'effet d'une erreur. Au vu des textes lui ayant été soumis, le comité de la revue a jugé bon de déroger aux règles habituelles. Cela lui permet de consentir, exceptionnellement, aux auteurs sélectionnés un petit peu plus d'espace qu'à l'ordinaire.

Par souci d'équilibre, nous avons retenu deux textes qui sont résolument de nature empirique et deux textes plus spéculatifs.

Sur le plan théorique, il nous a paru utile de retenir des articles présentant des perspectives différentes. L'exclusivité du propos n'a en effet été concédée, dans ce numéro, ni aux approches dites du passage à l'acte, ni à celles de la réaction sociale. 
Si nous avons préféré à l'appellation de « criminalité des affaires » celle de «criminalité économique », c'est parce que nous ne soubaitons pas nous restreindre à la criminalité des animateurs de l'économie privée. Nous entendons désigner ainsi l'ensemble des crimes contre les biens qui sont commis sans violence, mais par quelque astuce ou tromperie ou encore par l'abus d'une position de puissance ou d'influence, en vue d'un profit. Nous ne visons pas ici les infractions commises dans le contexte d'activités ou d'organisations illicites par nature, mais uniquement des activités se déroulant dans un contexte, une structure, des entreprises fondamentalement légitimes et conformes à l'ordre juridique.

C'est là une notion du crime économique qui s'inspire largement des définitions classiques d'auteurs comme Reiss et Biderman ou Sparks ${ }^{3}$. C'est aussi une définition fort proche de celle que, dans sa contribution, Laureen Snider nous propose du crime en col blanc. Le White-Collar Crime ayant toutefois été défini pour la première fois par Sutherland, il nous semble nécessaire de prendre nos distances d'avec l'appellation. La raison en est que Ja notion sutherlandienne était fort restrictive, puisque le crime en col blanc était, par définition l'apanage des membres des hautes sphères de la société. Aussi le souci d'échapper à cette restriction nous conduit-il à préférer la notion, plus vaste, de crime économique.

Les textes retenus contribuent d'ailleurs à démontrer qu'outre la criminalité économique de haut vol, il existe une criminalité économique "démocratique " qui reste à la portée d'individus sans qualité ou position exceptionnelle et qui est, de fait, pratiquée par un large éventail de délinquants.

Dans cette matière, les sociétés, qu'elles soient publiques ou privées, tiennent une place de choix. Si ce n'est celui-là même d'auteur du crime, les entreprises peuvent également jouer le rôle d'instrument du délit (d'écran) ou encore celui de victime. Les articles retenus donnent d'ailleurs un aperçu de la pluralité des implications « corporatives » en matière de crimes économiques.

C'est en particulier l'article de L. Snider qui traite le plus abondamment des problèmes que pose la délinquance des entreprises. Sans nullement se cacher de ses convictions et de ses préférences idéologiques, L. Snider

3. A. J. REISS/A.D.BEIDERMAN, Data Sources on White-Collar Law Breaking, Washington, D.C., NIJ, U.S. Department of Justice, 1980, pp. 4 et ss.

R. F. SPARKS, "White Collar Crime : The Problem and the Federal Response», in: Hearings before the Subcommittee on Crime of the Committee of the Judiciary House of Representatives, 95th Congress. Washington, D.C., U.S. Government Printing Office, 1979 , pp. 166 et ss. 
dénonce la dérégulation de l'économie. Déplorant l'étroite interdépendance existant actuellement entre l'économie privée et l'État, elle constate que celuici est de moins en moins à même d'infliger des sanctions aux entreprises.

L. Snider relève en particulier à quel point la réglementation et le contrôle par l'État des activités dangereuses et dommageables des entreprises sont remis en question par les théories dites du risk management. Elle nous livre enfin bien des raisons de regretter que les gouvernements nord-américains lâchent toujours davantage la bride des entreprises.

Compte tenu de la mondialisation galopante des échanges économiques et de la criminalité à laquelle elle donne lieu, il nous a paru utile de retenir un article qui évoque la dimension internationale de certaines formes de la criminalité économique, en l'occurrence le blanchiment d'argent. Dans ce texte, Michael Levi démontre en particulier à quel point il est difficile pour la communauté internationale d'arrêter, en matière de blanchiment une politique criminelle concertée susceptible d'être appliquée dans des contextes nationaux très différents et de présenter le même ratio avantages/ inconvénients pour chacun des États participants. L'auteur évoque en outre l'importance que revêt la collaboration du secteur privé, notamment des entités corporatives bancaires et financières, en matière de lutte contre le blanchiment.

Marc Alain présente une activité criminelle lucrative : la contrebande des produits du tabac. On peut d'abord douter qu'elle mérite d'être assimilée à de la criminalité économique au sens où nous l'avons définie. S'il est vrai qu'une forte proportion de contrebandiers n'ont que des activités illicites et qu'à cet égard ils ne sont pas partie prenante de l'économie légitime, il demeure que les contrebandiers dépendent très directement des fabricants de cigarettes. À cet égard, les contrebandiers permettent en fait à ces acteurs officiels de l'économie que sont les fabricants de cigarettes de satisfaire le plus grand nombre possible de consommateurs, surtout ceux pour qui les taxes sur la cigarette constituent une entrave.

M. Alain fait d'ailleurs très utilement la différence entre l'offre de cigarettes de contrebande et la demande, laquelle dépend notamment du gouvernement et des ses politiques fiscales, mais aussi des rapports entre le citoyen et l'État.

Alors que le sujet traité par M. Alain a une dimension internationale, le texte de Luc Vallée et Stéphane Dupuis porte sur une criminalité clairement circonscrite dans l'espace urbain de Montréal. Il s'agit en l'occurrence de fraude à l'assurance et, pour être précis, de fraude à l'assurance par incendie volontaire. Si cette criminalité peut s'avérer menaçante pour la santé ou la vie d'autrui et qu'elle peut donc causer des dommages autres que matériels, 
elle reste avant tout motivée par la recherche d'un gain économique. Le lien entre la fraude à l'assurance et les activités commerciales légitimes est évident dans la mesure où, mis à part les cas minoritaires où le fraudeur prend une police d'assurance dans le but de frauder, le fraudeur à l'assurance abuse d'un contrat auquel il a préalablement adhéré sans arrière-pensée.

Le choix des contributions que nous avons fait ici n'est certes pas de nature à rendre compte de toute l'étendue du champ sur lequel nous nous engageons. Il peut toutefois nous en donner un aperçu, illustrer la diversité des criminalités que l'on peut qualifier d'économiques et nous offrir un petit échantillon des différentes approches méthodologiques pouvant être mises à profit dans ce champ. Il nous a enfin permis d'accorder à la production criminologique empirique une part respectable de ce numéro.

Mais trêve de considérations préliminaires et place au sujet ! 